\title{
Content-aware Image Retargeting Based on Visual Effect Assessment
}

\author{
Lihua $\mathrm{Bi}^{1}$ and Canlin $\mathrm{Li}^{2}$ \\ ${ }^{1}$ Office of Academic Affairs, Zhengzhou University of Light Industry, Zhengzhou \\ 450000, China \\ ${ }^{2}$ School of Computer and Communication Engineering, Zhengzhou University of \\ Light Industry, Zhengzhou 450000, China \\ lcl_zju@aliyun.com
}

\begin{abstract}
Content-aware image retargeting has drawn much attention in image and vision research in recent years. However, existing methods are very difficult to ensure that the result images from retargeting achieve good visual effect on the whole, since these methods mainly focus on spatial image information. In this paper, we propose a new approach on content-aware image retargeting based on visual effect assessment. We establish an evaluation mechanism of the visual effects of retargeted images which is based on a priori statistical knowledge through studying the user's evaluation, and build the computable model of visual effect assessment of retargeted image with the help of mathematical description from Dynamic Bayesian Networks. After finishing contentaware processing and construct a three-level model of visual saliency contents for the original image, we retarget the original image into the target image by virtue of deforming image, and integrate computable model of visual effect assessment into retargeting process, so as to guide the retargeting. Finally, by steadily adjusting the size of the intermediate results from deforming image, we make this size be eventually equal to the size of the target image of retargeting, under the constraint that the result image should acquire good visual effect through optimizing the parameters of visual effect assessment. Real data have been used to test the proposed approach and very good results have been achieved, validating it.
\end{abstract}

Keywords: Content-aware, Image Retargeting, Visual Effect Assessment, Deformation, Feedback-based

\section{Introduction}

Image retargeting has drawn much attention in image and vision research in recent years. Retargeting images for display on devices with different resolutions and aspect ratios is an important problem for the modern society, where visual information is accessed using a variety of display media with different formats, such as cellular phones, PDAs, widescreen television, and more. This problem further increases with the explosion of image and video content on the web. To address this problem, content-aware retargeting was recently introduced. Content-aware image retargeting is a technique that adjusts input images into arbitrary sizes and simultaneously preserves the salient regions of the input images.

Content-aware image retargeting methods can be classified as discrete or continuous [1]. Discrete methods regard images as collections of pixels and decide which pixels to remove (or duplicate) in order to obtain the target aspect ratio. Cropping techniques [2-5] cut rectangular regions adjacent to the image boundary while trying to avoid removing salient objects. Seam carving [6,7] removes or duplicates contiguous but not necessarily 
straight chains of pixels that pass through homogeneous regions of the image. Some recent works employ patches instead of individual pixels and preserve patch coherence between the source and target images, enabling automatic removal of repetitive patterns [8-10]. The shift-map method [11] removes entire objects at a time, alleviating the discontinuity artifacts of pixel-based carving. Continuous retargeting methods use variational formulation to design warping functions, such that the shape of salient regions is preserved while homogeneous regions are squeezed or stretched. Gal et al. proposed an approach that warps an image into an arbitrary shape while preserving user-specified features [12]. The feature-aware warping is achieved by a particular formulation of the Laplacian editing technique, suited to accommodate similarity constraints on parts of the domain. Wolf [13], et al., developed a method that assigns a saliency score to each pixel in the image. An optimized transformation of the video to a downsized version is then calculated that respects the saliency score. The transformation shrinks less important regions more than important ones. Wang, et al., [14] presented a "scale-and-stretch" warping method that allows resizing images into arbitrary aspect ratios while preserving visually prominent features. The method operates by iteratively computing optimal local scaling factors for each local region and updating a warped image that matches these scaling factors as closely as possible. The amount of deformation of the image content is guided by a significance map that characterizes the visual attractiveness of each pixel; this significance map is computed automatically using a novel combination of gradient and salience-based measures. Zhang, et al., [15] proposed pre-computing a per-pixel cumulative shrinkability map which takes into account both the importance of each pixel and the need for continuity in the resized result. A random walk model is used to find the shrinkability of each pixel, leading to a linear system which can be solved by a multi-grid solver, allowing for efficient implementation, which in principle could readily be adapted for use on a GPU. All image retargeting methods have the fundamental spatial limitation: if there is not enough unimportant content in the image, salient objects must be distorted or removed.

Most content-aware retargeting techniques to date have concentrated on spatial image information, such as various visual saliency measures and object detection, to define visually important parts of the image and to guide the retargeting process. They rely on the fact that removing or distorting homogeneous background content is less noticeable to the eye [1]. However, these methods are very difficult to ensure that the result images from retargeting achieve good visual effect on the whole. Actually, the retargeted images are eventually evaluated and used by the users. There is a general consensus among people regarding the evaluation of retargeting results. For example, the users consistently demonstrate high sensitivity to deformation, particularly for images that include specific types of content like faces, well defined geometric structures and symmetry. If we can establish an evaluation mechanism of retargeted images and their visual effects which imitates the user's evaluation, and apply this mechanism to retargeting processing so as to optimize the retargeting, the result images will probably get good visual effects. From the above idea, we propose a content-aware image retargeting based on visual effect assessment in this paper. We try to assess the visual effects of retargeted image based on a priori statistical knowledge, and build the computable model to assess the visual effects of retargeted image with the help of mathematical description from Dynamic Bayesian Networks. After finishing content-aware processing and construct a three-level model of visual saliency contents for the original image, we retarget the original image into the target image by virtue of deforming image, and integrate computable model of visual effect assessment into retargeting process. This also makes the proposed approach on content-aware image retargeting actually become a kind of feedback-based approach for retargeting. Finally, by steadily adjusting the size of the intermediate results from deforming image, we make this size be gradually close to and eventually equal to the size 
of the target image of retargeting, under the constraint that the image after deformation should acquire good visual effect through optimizing the parameters of visual effect assessment.

The remainder of this paper is organized as follows. Section 2 illustrates the framework of the proposed approach on content-aware retargeting based on visual effect assessment. Section 3 explores how to assess the visual effects of retargeted image and build the computable model to assess the visual effects of retargeted image. Section 4 elaborates the specific implementation of the proposed approach on content-aware retargeting based on visual effect assessment. Section 5 provides the experimental results. Finally, the paper is concluded in Section 6.

\section{Framework of the Proposed Approach on Content-aware Retargeting}

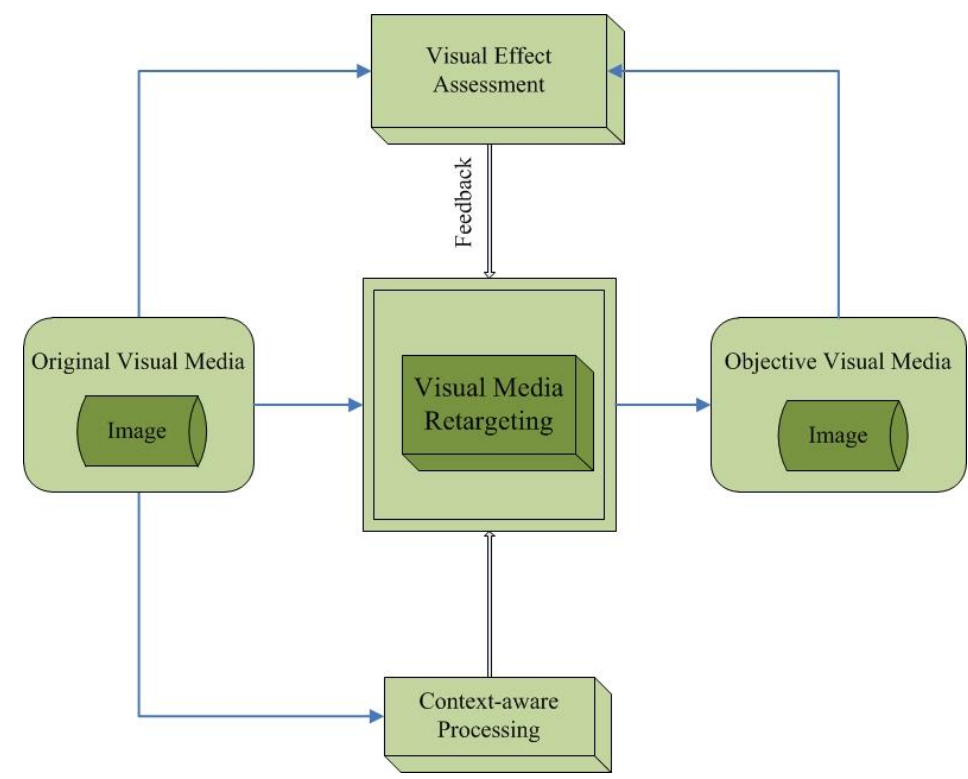

Figure 1. Schematic Diagram of the Proposed Approach on Content-aware Image Retargeting based on Visual Effect Assessment

Figure 1 shows the schematic diagram of the approach on content-aware image retargeting based on visual effect assessment proposed by us in this paper.

As we can see from Figure 1, the proposed content-aware image retargeting based on visual effect assessment mainly involves the following steps. First of all, the contentaware processing is conducted in the original image, and makes us distinguish between the visual salient contents and non-salient contents in the image, and build a three-level model of visual saliency contents for the image. Then, we explore the users' evaluation mechanism on visual effects of retargeted image, and build the computable model to assess the visual effects of retargeted image with the help of mathematical description from Dynamic Bayesian Networks. After that, we retarget the original image toward target image by virtue of deforming on it, under the constraint of keeping the resulting image having good visual effects. At last, by progressively trimming or stretching the original image, we make the size of deformed image be eventually consistent with the size of the target image of retargeting. Hereinafter we will show the detailed procedure of the proposed approach by us. 


\section{Visual Effect Assessment}

As for visual effect assessment, we propose an approach to assess the visual effects of retargeted image based on a priori statistical knowledge. From the cognitive mechanism, by using the method of behavioral experiments, we explore the users' evaluation mechanism on visual effects of retargeted image, and build a computable model to assess the visual effects of retargeted image. Figure 2 shows the diagram of the proposed approach on visual effect assessment. The details are as follows. Depending on the evaluation mission of desired visual effects, we acquire some experimental materials on retargeted images by virtue of the existing retargeted methods such as [6, 7]. These retargeted images involve some specific visual effects, which will lead the users to evaluate the experimental materials from the visual aspect. We can obtain the visual effect assessment of users on the retargeted images, and extract the parameters of visual effect assessment, and analyze the relationship between the visual effect assessment and retargeted image and its specific visual effect from the statistical point of view. Specific visual effects include distortion of geometric structure, distortion of proportion, removal of content, over-squeezing and over-stretching, etc. Through analyzing the changes of user's evaluation caused by appearance or absence of some specific visual effect, we focus on exploring the relationship between spatial and temporal variations on the parameters of visual effect assessment and specific visual effect. Finally we build the computable model to assess the visual effects of retargeted image with the help of mathematical description from Dynamic Bayesian Networks.

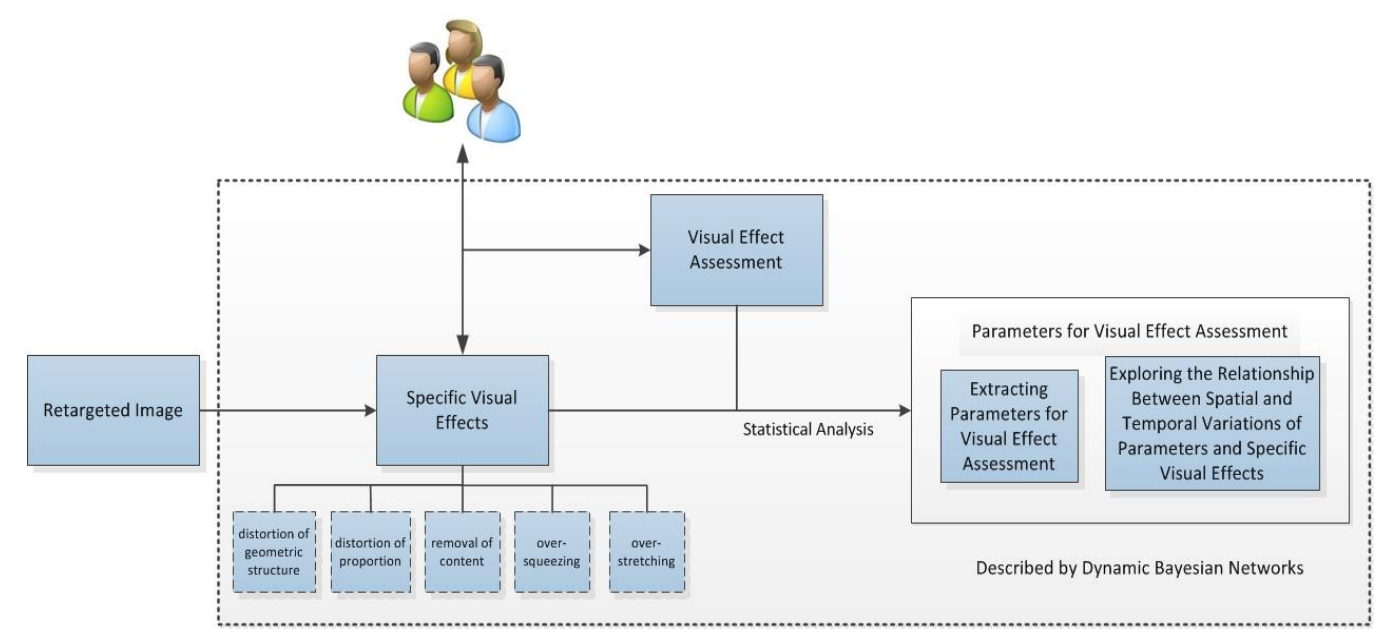

Figure 2. Diagrammatic Sketch of Visual Effect Assessment for Retargeted Images

\section{Content-aware Image Retargeting Based on Visual Effect Assessment}

This paper proposes an approach on content-aware image retargeting based on visual effect assessment, which attempts to integrate the evaluation of retargeting results into retargeting process, so as to optimize the results of retargeting.

After being retargeted, the objective image is often used to some ordinary occasions by the users, so the retargeted image is undoubtedly and eventually evaluated by the users. There is a general consensus among people regarding the evaluation of retargeting results. For example, the users consistently demonstrate high sensitivity to deformation, particularly for images that include specific types of content like faces, well defined geometric structures and symmetry. In many cases users prefer sacrificing content over 
inserting deformation to the media. The findings like these are invariant to whether or not the users are aware of the original image. So the evaluation of retargeting results mainly focuses on visual effect assessment of retargeted images. Thus, we may model the quality of a retargeted result by mathematical and statistical analysis and evaluate the result directly. In the previous section, we have built the computable model to assess the visual effects of retargeted image. Here we need to integrate computable model of visual effect assessment into retargeting process. The model can be used to guide the retargeting process, optimizing the retargeting results. This makes the proposed approach on contentaware image retargeting actually become a kind of feedback-based approach for retargeting.

We have illustrated the schematic diagram of the proposed approach on content-aware image retargeting based on visual effect assessment in Figure 1, and the diagrammatic sketch of visual effect assessment for retargeted images in Figure 2. Figure 3 demonstrates the specific implementation of the proposed approach on content-aware image retargeting, which includes the combination of visual effect assessment into retargeting process. Specific details are described below.

In the first stage, we conduct the content-aware processing for the original image, and make a clear distinction between the salient contents and non-salient contents in the image, and build visual saliency map for the original image. The existing saliency maps only focus on considering local and low-level features of media contents, making them difficult to meet the real media semantics. Here when we construct visual saliency map, we detect visual salient regions in the low-feature level as well as in the object and semantic unit level, and establish a three-level model of content importance from low to high involving low-level feature, high-level feature and visual semantic unit. Through the arrangement and combination of three levels, we make an optimization of the saliency map, in order to truly capture the important visual contents which meet media semantics. We need to keep the visual salient regions as possible as intact after retargeting.

Next, we retarget the original image into the target image by making the deformation on the original image and evaluating the visual effect of the result image. During this processing, we try to preserve the visual salient contents, deform the less important contents and smooth the deformation and intensity of overlapped regions. We use the grid-based representation to describe the less important regions, so as to conduct constrained deformation on them and ensure that the boundaries of these regions conform to the boundaries of visual salient regions. Graph-cut is also used to minimize the discontinuity of intensity of overlapped regions. When the deformation is conducted, we keep on evaluating the visual effect of the result image and optimizing the parameters of visual effect assessment.

In the end, by steadily adjusting the size of the intermediate results from deforming image, we make this size be gradually close to and eventually equal to the size of the target image of retargeting, under the constraint that the deformed result should be consistent with objective function and the image after deformation should acquire good visual effect through optimizing the parameters of visual effect assessment. 


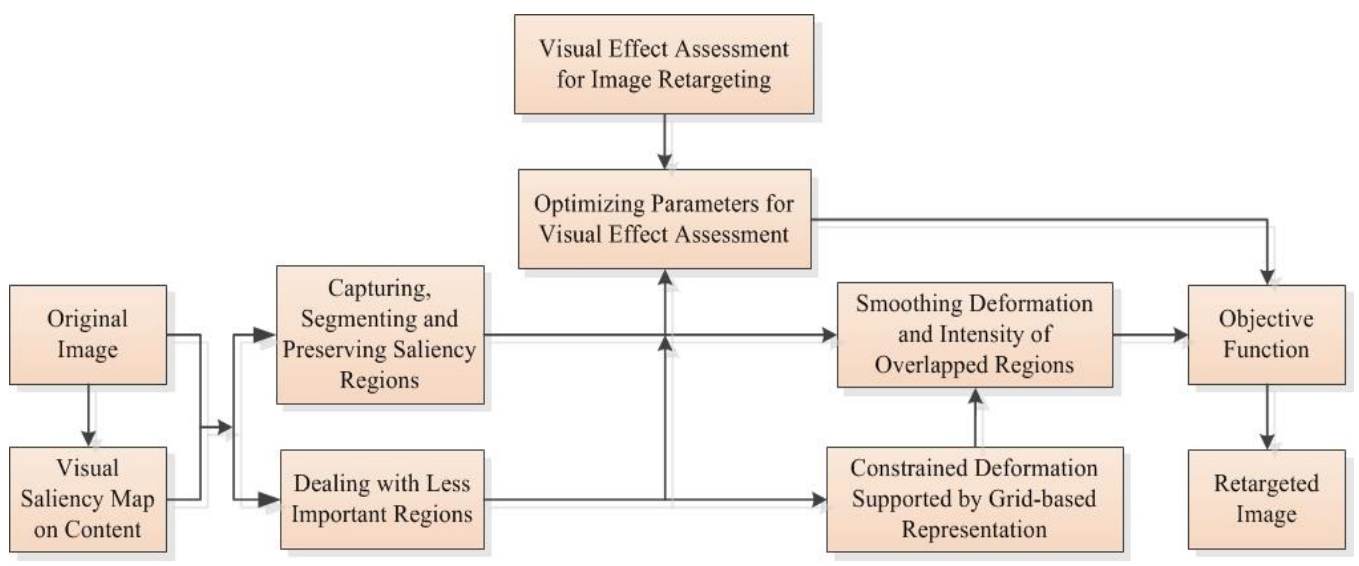

Figure 3. Illustration of the Implementation on the Proposed Approach of Content-aware Image Retargeting based on Visual Effect Assessment

\section{Experimental Results}

To validate the proposed approach on content-aware image retargeting based on visual effect assessment in the paper, we took real images to confront it with the real world in this section. The image dataset in our experiment is slected from the RetargetMe dataset [16] which contains dozens of original images with various attributes. Each original image has all or partial of eleven retargeted images generated by the existing retargeting techniques such as seam carving (SC), shift-map (SM), non-homogeneous warping (WARP), scale-and-stretch (SNS), and so on, respectively, through shrinking or stretching the original image to different degrees. We also compare the retargeting results obtained from our method with the results from some existing retargeting techniques.

As shown in Figures. 4-6, we select nine typical cases of image similarity measure for retargeting in the paper due to space limitation, which involve different scenes including "blueman", "child", "face", "Johanneskirche", "greek_wine", "Umdan", "car", "islands" and "painting2" for illustrating the extensive application of our method. According to the degree with original image being shrunk or stretched during retargeting process, we divided them into three groups. As illustrated in Figure 4 from top to bottom, the first group involves four cases: "blueman", "child", "face" and "Johanneskirche", where the original images have been shrunk into retargeted images with a ratio of 0.75 in width. As shown in Figure 5 from top to bottom, the second group involves two cases: "greek_wine" and "Umdan", where the original images have been shrunk with a ratio of 0.5 in width, with being narrower. As illustrated in Figure 6 from top to bottom, the third group involves three cases: "car", "islands" and "painting2", where the original images have been stretched with a ratio of 1.25 in width.

We retarget the original image in the leftmost of every case into the targeted size using our proposed content-aware image retargeting approach. Specifically, we conduct the content-aware processing for the original image, and make a clear distinction between the salient contents and non-salient contents in the image. Then we retarget the original image toward target image by virtue of deforming on original image and evaluating the visual effect of the result image. According to Section 4, by virtue of progressively trimming or stretching the original image and steadily adjusting the size of the intermediate results from deforming image, we make the size of deformed image be eventually consistent with the size of the target image of retargeting, under the constraint that the deformed result should be consistent with objective function and the image after deformation should acquire good visual effect through optimizing the parameters of visual effect assessment. We also made a comparison between the retargeting results obtained from our approach and the results from other existing retargeting techniques. As shown in Figures 4-6, the 
remaining images in each case are generated by seam carving (SC) [7], shift-map (SM) [11], non-homogeneous warping (WARP) [13], scale-and-stretch (SNS) [14] and our approach from left to right, respectively.

It is mainly because of poor visual effects to make the retargeted result be rejected by users. Poor visual effects often involve distortion of geometric structure, distortion of proportion, removal of content, over-squeezing and over-stretching about visual salient regions, etc. These poor visual effects also make the retargeted image have poor similarity to the original image. According to the obtained results in Figures 4-6, we can figure out that the retargeted image generated by our proposed approach involves few of these poor visual effects of visual salient regions. From these nine cases, on the whole, the retargeted images generated by our method generally achieve good visual effects as well as good similarity to the original image. Compared with other methods, the results obtained by our method are more easily accepted by the subjective human choices, from the perspective of visual effect assessment on the retargeted image. Thus our proposed method about content-aware image retargeting based on visual effect assessment has been validated.
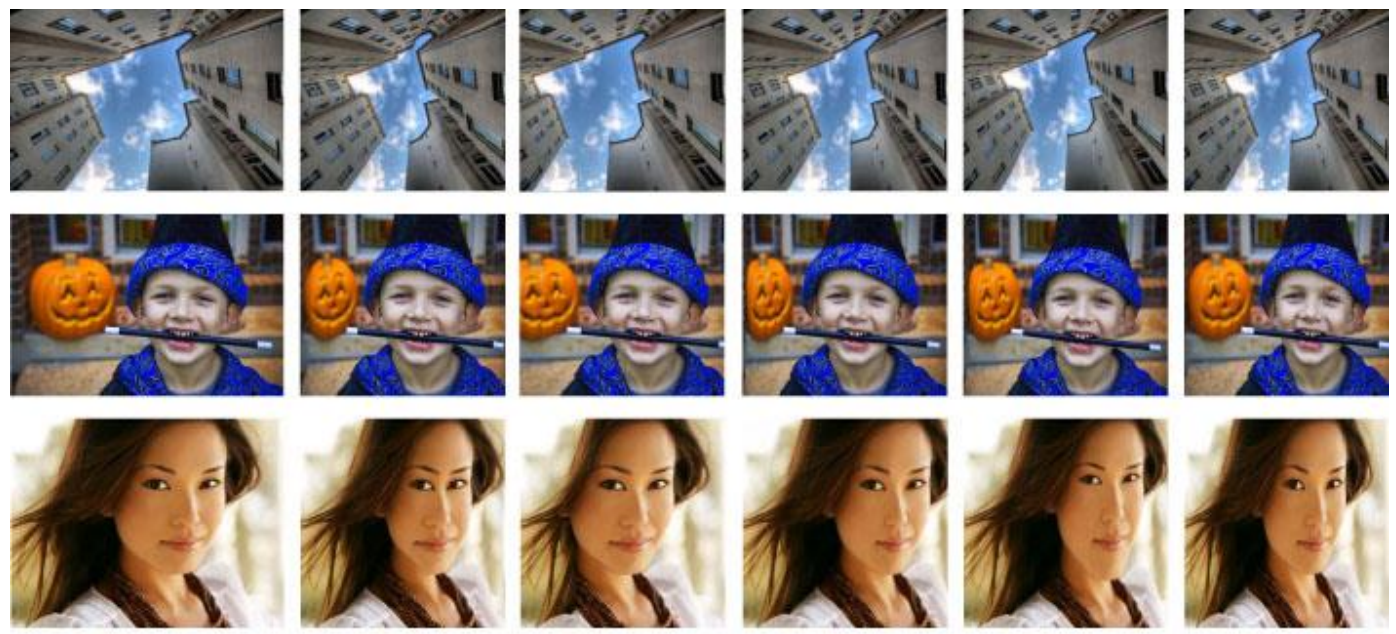

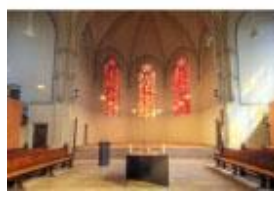

(a)

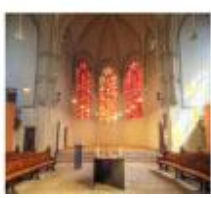

(b)

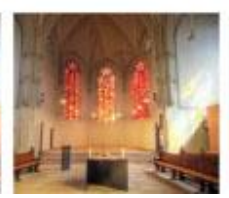

(c)

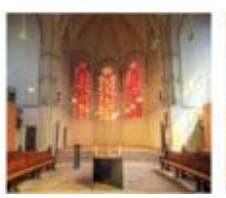

(d)

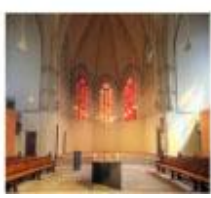

(e)

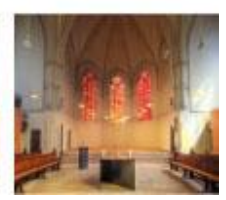

(f)

Figure 4. The First Group of Image Retargeting Cases where the Original Images Have Been Shrunk Into Retargeted Images with a Ratio of 0.75 in Width. From Top to Bottom, these Cases are "Blueman", "Child", "Face" and "Johanneskirche", and from Left to Right, these Images are (a) the Original Image, and the Retargeted Image Generated by (b) SC, (c) SM, (d) WARP, (e) SNS and (f) Ours 


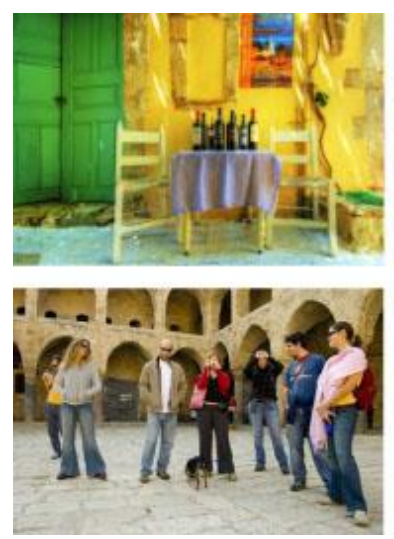

(a)
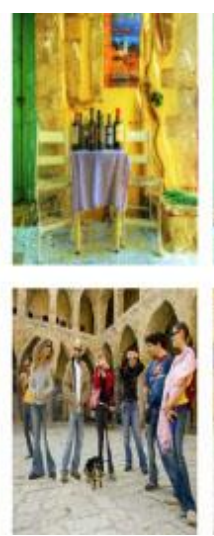

(b)

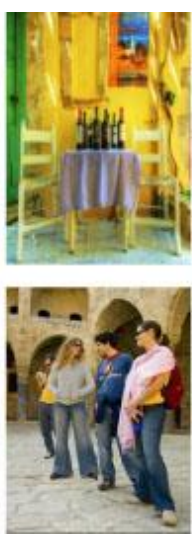

(c)
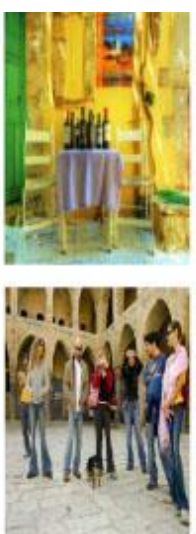

(d)

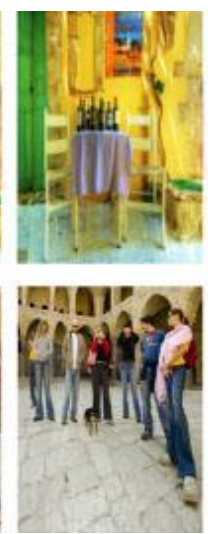

(e)
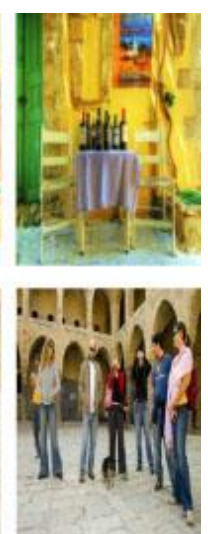

(f)

Figure 5. The Second Group of Image Retargeting Cases where the Original Images have been Shrunk into Retargeted Images with a Ratio of 0.5 in Width. From Top to Bottom, these Cases are "greek_wine" and "Umdan", and from Left to Right, these Images are (a) the Original Image, and the Retargeted Image Generated by (b) SC, (c) SM, (d) WARP, (e) SNS and (f) Ours
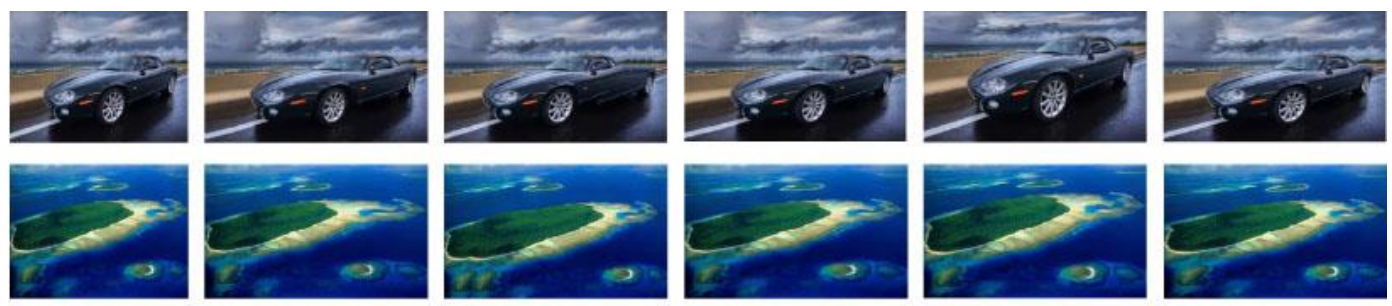

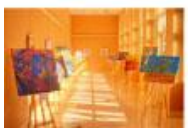

(a)

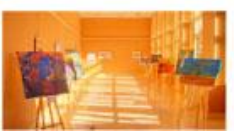

(b)

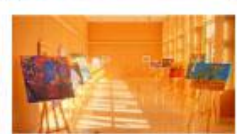

(c)

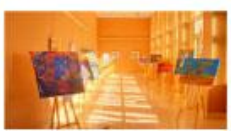

(d)

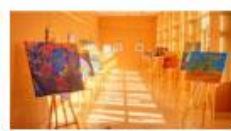

(e)

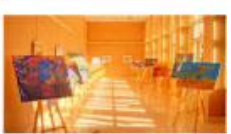

(f)

Figure 6. The Third Group of Image Retargeting Cases where the Original Images have been Shrunk into Retargeted Images with a Ratio of 1.25 in

Width. From Top to Bottom, these Cases are "Car", "Islands" and

"Painting2", and from Left to Right, these Images are (a) the Original Image, and the Retargeted Image Generated by (b) SC, (c) SM, (d) WARP, (e) SNS and (f) Ours

\section{Conclusion}

In this paper, we proposed a novel approach on content-aware image retargeting based on visual effect assessment. The proposed approach on content-aware image retargeting mainly includes the following steps. Firstly, we conduct the content-aware processing for the original image, and make a clear distinction between the salient contents and nonsalient contents in the image, and build a three-level model of visual saliency contents for the original image, so as to truly capture the important visual contents which meet media semantics. Then, we establish a mechanism of visual effect assessment of retargeted images which imitates the user's evaluation and assess the visual effects of retargeted image based on a priori statistical knowledge, and build the computable model of visual effect assessment of retargeted image. Next, we retarget the original image into the target image by virtue of deforming image, and apply computable model of visual effect assessment to retargeting processing so as to optimize the retargeting. At last, by steadily trimming or stretching the original image and keeping the deformed image to be as 
possible as intact from visual saliency contents, we make this size of the deformed image be eventually consistent with the size of the target image of retargeting, under the constraint that the image after deformation should achieve good visual effect through optimizing the parameters of visual effect assessment. Experimental results showed that the achieved content-aware retargeting results for real images by our approach generally achieve good visual effects as well as good similarity to the original image. Compared with other techniques, the results obtained by our method are more easily accepted by the subjective human choices, from the perspective of visual effect assessment of the retargeted image. The good results from the experiments illustrated the practicability and effectiveness of our proposed content-aware retargeting approach, validating it.

\section{ACKNOWLEDGEMENTS}

The work is supported by the National Nature Science Foundation of China (No.U1404623), the Natural Science Foundation of Henan Educational Committee (No.12B520068) and the PHD Foundation of Zhengzhou University of Light Industry (No.2011BSJJ002)

\section{References}

[1] A. Shamir and O. Sorkine, "Visual media retargeting", In ACM SIGGRAPH ASIA 2009 Courses, (2009) December 16-19, Yokobama, Japan.

[2] L. Chen, X. Xie, X. Fan, W. Ma, H. Zhang and H. Zhou, "A visual attention model for adapting images on small displays", Multimedia systems, vol. 9, no. 4, (2003), pp. 353-364.

[3] H. Liu, X. Xie, W. Y. Ma and H. J. Zhang, "Automatic browsing of large pictures on mobile devices", In: Proceedings of the eleventh ACM international conference on Multimedia, (2003) November 2-8, Berkeley, CA, USA.

[4] B. Suh, H. Ling, B. B. Bederson and D. W. Jacobs, "Automatic thumbnail cropping and its effectiveness", In: Proceedings of the 16th annual ACM symposium on User interface software and technology, (2003) November 2-5, Vancouver, Canada.

[5] A. Santella, M. Agrawala, D. Decarlo, D. Salesin and M. Cohen, "Gaze-based interaction for semiautomatic photo cropping", In: Proceedings of the SIGCHI conference on Human Factors in computing systems, (2006) April 22-27, Montréal, Québec, Canada.

[6] S. Avidan and A. Shamir, "Seam carving for content-aware image resizing", ACM Transactions on Graphics, vol. 26, no. 3, (2007), pp. 10.

[7] M. Rubinstein, A. Shamir and S. Avidan, "Improved seam carving for video retargeting". ACM Transactions on Graphics, vol. 27, no. 3, (2008), pp. 16.

[8] T. S. Cho, M. Butman, S. Avidan and W. T. Freeman, "The patch transform and its applications to image editing", In IEEE Conference on Computer Vision and Pattern Recognition, (2008) June 24-26, Anchorage, Alaska, USA.

[9] D. Simakov, Y. Caspi, E. Shechtman and M. Irani, "Summarizing visual data using bidirectional similarity", In: IEEE Conference on Computer Vision and Pattern Recognition, (2008) June 24-26, Anchorage, Alaska, USA.

[10] C. Barnes, E. Shechtman, A. Finkelstein and D. B. Goldman, "PatchMatch: A randomized correspondence algorithm for structural image editing”, ACM Transactions on Graphics, vol. 28, no. 3, (2009), pp. 24.

[11] Y. Pritch, E. Kav-Venaki and S. Peleg, "Shift-map image editing", In IEEE International Conference on Computer Vision, (2009) September 29-October 2, Kyoto, Japan.

[12] R. Gal, O. Sorkine and D. Cohen-Or, "Feature-aware texturing", In Proceedings of the 17th Eurographics conference on Rendering Techniques, (2006) June 26-28, Nicosia, Cyprus.

[13] L. Wolf, M. Guttmann and D. Cohen-Or, "Non-homogeneous content-driven video-retargeting", In IEEE 11th International Conference on Computer Vision, (2007) October 14-20, Rio de Janeiro, Brazil.

[14] Y. S. Wang, C. L. Tai, O. Sorkine and T. Y. Lee, "Optimized Scale-and-Stretch for Image Resizing", ACM Transactions on Graphics, vol. 27, no. 5, (2008), pp. 118. 
[15] Y. F. Zhang, S. M. Hu and R. R. Martin, "Shrinkability maps for content-aware video resizing”, In: The 16th Pacific Conference on Computer Graphics and Applications (Pacific Graphics) (2008) October 810, Tokyo, Japan.

[16] "RetargetMe", http://people.csail.mit.edu/mrub/retargetme/.

\begin{abstract}
Authors
Li Canlin, received $\mathrm{PhD}$ degree in computer science from Shanghai Jiaotong University in 2010. Since 2010 he has been in the School of Computer and Communication Engineering at Zhengzhou University of Light Industry. His research interests include image processing, multimedia, graphics, digital entertainment and software engineering. Dr. Li is a member of IEEE as well as ACM.
\end{abstract}

